\title{
Rebyu ng Pagpasok sa Eksena: Ang Sinehan sa Panitikan at Pag-aaral ng Piling Sinehan sa Recto ni Chuckberry J. Pascual
}

Glenn L. Diaz

Hindi bago, kung tutuusin, ang interdisciplinary na lapit sa pagsusuri ng espasyo't kultura. Halimbawa, maraming pag-aaral tungkol sa mga katutubong grupo ang nakabatay sa tagpuan ng kanilang panitikang oral at materyal na mga kondisyon. May ilan ring pagninilay ukol sa mga partikular na bayan o sa ideya ng "lungsod" na pawang sumasangguni sa dalawang "teksto."

Sa Pagpasok sa Eksena: Ang Sinehan sa Panitikan at Pag-aaral ng Piling Sinehan sa Recto, ginawang "lunsaran" ni Chuckberry Pascual (2016) para sa kaniyang etnograpiya ng mga sinehan sa Maynila ang mga tekstong pampanitikan na tumatampok sa sinehan bilang lunan ng gawaing homoseksuwal. Paliwanag niya, mistulang "mapa" ang mga teksto dahil sa "makatotohanan" at "siyentipiko" na paglalahad ng karanasan ng mga baklang manunulat, ideya na unang isinulong ni J. Neil Garcia, isa sa mga patnugot ng Ladlad anthology series, na pinagmulan ng marami sa mga akdang kanyang sinuri.

Mahalaga ang pasintabing ito. Maaaring pulaan ang pag-aaral dahil, kaiba sa mga pangkat etniko, ang mga "katutubo" ng mga sinehan ay walang organikong koneksiyon sa pag-iral ng mga akda liban sa karanasang itinatampok. Susog pa ni Pascual, "dinamiko" at hindi "patag" ang relasyong nabubuo sa pagitan ng tekstong pampanitikan at espasyong homoseksuwal, at ang mismong akto ng pagsusulat ay nagsisilbing pagsasalehitimo sa huli. 
Sa kabuuan ay naging mabunga ang taktikang ito. Gamit ang "mapa," masinsing natukoy ni Pascual ang iba't ibang praktika at "talinghaga" sa mga akda at matapos ay itinanghal ang kaukulang etnograpikong patunay. Ilan sa mga ito ang pagrampa, paghahabulan, at pagtatalik sa loob ng sinehan at mga talinghaga ng dumi, dilim, entablado at pagtatanghal, at paglambot.

Matalas din ang pag-uugat ng mga nasipat sa loob sa kalagayan ng mga bakla sa "labas." Halimbawa, ang karumihan ng mga sinehan bilang espasyo, aniya, ay salungat sa "kalinisan" sa labas, na dulot ng pagmamatyag ng lipunang heteroseksista. May nakapanayam naman na inihalintulad ang sarili at mga parokyano kay Madonna, isang gay icon, patunay umano na mulat ang mga ito sa pagtatanghal na dimensiyon (performance o performativity) ng mga praktika sa loob ng sinehan, mula sa pagrampa hanggang sa mga linya na pawang "scripted."

Sa paraang ito rin nahinuha ni Pascual ang isang talinghaga na hindi niya nahanapan ng etnograpiyang katumbas: ang paglambot (tenderness). "Bagamat nababanggit ang pag-ibig ... tila ba isa itong masamang salita kapag nasa konteksto ng usapan tungkol sa sinehan" (Pascual, 2016, pp. 92-93). Sityo man ng "pangangailangan ng pag-ibig" (aking diin), sa huli'y walang pag-ibig ang hinahayaang umusbong sa karimlan ng espasyo, at walang ibang pakahulugan ang mga parokyano ng sinehan liban sa lugar kung saan may posibilidad ng pakikipagtalik (kung ang pakikipagtalik ay maituturing ding pag-ibig ay iba pang usapin).

Sa isang banda'y romantisismo ang marapat singilin sa anomalyang ito; ngunit pinatitingkad din ng rebelasyon ang waring nakaligtaan ni Pascual: ang porma ng mga akda. Bilang saligan ng modernong panitikan (at batayang pagkakaiba sa panitikang oral), porma ang naghahayag ng pagiging artifice ng mga tula at maikling kuwento, at sa akto ng pagkakalikha ng mga ito ay pinupuspos na ng magkakawing-kawing na pantasya mula sa indibidwal, sa halip na kolektibong paglalang. Salat, kung gayon, ang suri sa antas lamang ng tema; kung etnograpikong datos ang habol sa mga akda-sa mga tula, lalo na-lumalabas na hindi ganap na panitikan ang turing sa mga piyesa.

Hindi nito pinawawalang-bisa ang mga ipinunto ng pag-aaral; utang, kung tutuusin, sa mapang inilatag ng mga tesktuwal na akda ang masinsing taksonomiya ng mga nabanggit na praktika at talinghaga sa mga sinehan. Dahil sa mapang ito'y naging maingat, mayabong, at makabuluhan ang naging paraan ng pagsiyasat sa espasyo. Kung ituturing na sinehan, maalwan at maliwanag na lobby ang mapang ito.

Naging maalwan rin dahil kongkreto ang pundasyon. Isa pang angat na kalakasan ng Pagpasok sa Eksena ang atensiyon sa pagbubuo ng argumentong nakasandig sa malinaw na framework at konteksto. Mula sa mga inaasahang boses, tulad nina Judith Butler, Henri Lefebvre, at Michel 
Focault, hanggang kina Benedict Anderson, Caroline Hau, at Slavoj Zizek, matalas ang pakikipagniig ni Pascual sa mga ideyang sinangguni, at maingat ang paghimay at pagtagni (synthesis) ng kanilang mga pangunahing ambag sa kaniyang pagtatasa.

May ganito ring ingat at lawak sa kaniyang pagbaybay sa kasaysayan ng sinehan sa Pilipinas. Lagi't lagi, may tuon sa mga nakapangyayaring puwersa sa lipunan-pampolitika, pang-ekonomiya-na lumalang at patuloy na lumalalang sa pisikal na espasyo. Matagumpay na naiugnay, halimbawa, ang pag-iral ng mga nagsasariling sinehan sa mga isyung pang-media, gaya ng piracy, censorship, at nagbabagong nosyon ng "kalaswaan," at ibang kakawing na isyung panlipunan, tulad ng konsumerismo (pagsulpot ng mga mall, pag-usbong ng "pink spending") at mga institusyonal na aparato at simbulo (simbahan, MTRCB, Bagong Lipunan ni Marcos).

May sistematiko ring pahiwatig sa mga tatahaking landas. Halimbawa, pruweba umano ng maagang pagiging seksuwalisadong espasyo ng sinehan ang pagkahuli sa isang batang babaeng "nagbebenta ng aliw" sa harapan ng isang teatro ng mga Tsino sa Binondo noong 1862, kuwentong babalikan sa paliwanag sa "talinghaga ng dilim" sa susunod na kabanata. Sa anumang bahagi, buo ang aklat sa isip ni Pascual.

Bagaman hindi malalim na natalakay (at labas sa pakay ng pag-aaral), nagsilbi namang dagdag na dimensiyon ang paglilista sa mga pelikulang nakatulong sa "pagtatanghal ... sa homoseksuwalisasyong ng espasyo ng sinehan" sa ibang "tekstuwal" na paraan, gaya ng Tubog sa Ginto (Brocka, 1971), Maynila: Sa mga Kuko ng Liwanag (Brocka, 1975), at Tatay Kong Nanay (Brocka, 1978). Nabanggit din ang ebolusyon ng mga pelikulang tungkol sa bakla, tungo sa mas makabuluhan umanong Ang Lalaki sa Buhay ni Selya (Siguion-Reyna, 1997) o Pusong Mamon (Lamangan, 1998).

Napaigting naman ang katangiang meta ng ugnayang ito sa suri sa Serbis (Mendoza, 2008), na tungkol sa isang pamilya na nakatira sa isang lumang sinehan na ang pangalan ay Family. Samakatwid, "ang panonood ng Serbis sa Robinsons Galleria ay isang karanasang meta-espasyo" (Pacual, 2016, p. 53). Kahit heteroseksuwal at hanggang "ambient" lamang, waring pananggalang din ng mga parokyano ang mga pelikulang "malaswa" na ipinapalabas sa mga sinehan.

Dahil sa ganitong paghahanda, nang panahon na para pasukin ang mismong mga sinehan sa ikaapat na kabanata (ang pinakamahaba), may bitbit nang ideolohikal na bagahe ang espasyo. Imposible nang tingnan bilang dalisay at ilang ang anumang masisipat at maririnig sa loob, mula sa huntahan sa canteen hanggang sa graffiti sa banyo. Malinaw ang pag-inog ng lungsod at kasaysayan sa labas. Sa ganitong paraan naging artsibo ng mga personal at panlipunang dumi at salimuot ang mga sinehan. 
May parehong sinsin sa etnograpiyang pagsisiyasat sa loob, una sa mga indibidwal (empleyado, "pseudo-empleyado," parokyano, at kanilang mga interaksiyon, karaniwang gawi, huntahan, at iba pa) at ikalawa sa espasyo (labas ng sinehan, takilya, lobby, canteen, orchestra, at loge sections, palikuran, fire exit, at mga nosyon ng teritoryo). Mabusisi ang pagdedetalye sa mga transaksiyon na bumubuhay sa sinehan (na madalas ay walang direktang kinalaman sa mismong "palabas"), tulad ng dinamiko ng "pagrampa," uri ng gawaing sekswal, at presyong kaakibat ng mga "serbisyo."

Ginawaran din ng atensiyon ang mga nakapaskil na regulasyon ("Bawal gumawa ng kalaswaan sa loob ng sinehan"), graffiti sa banyo ("Dito lahat bakla panget pa"), pati ang himpilan ng radyo na pinakikinggan sa mga sinehan (Love Radio). Liban sa dagdag na paglalarawan sa espasyo, itinatanghal ng mga detalye ang mga tunggalian sa loob, na pawang kakabit ng mga natukoy nang kontradiksiyon sa labas, kasama na ang mga tangka ng mga "katutubo" na igpawan o makipamuhay sa mga ito.

Sa dulo, kung tunay na pawala na ang mga espasyong ito dahil sa Internet at pagsagka ng heteroseksistang lipunan, mahalagang piraso ng kasaysayan ng lungsod, ng Maynila, ang ambag ng pag-aaral. Sa totoo'y kasaysayan din ng "labas" ang ibinubunyag ni Pascual sa akto ng "pagpasok sa eksena," ang mga sala-salabit na ugnayang kasarian, materyal, at uri, at iba't ibang bersiyon ng eskrimahan ng at para sa kapangyarihan.

At kung sa huling kabanata ay inihambing sa "closet" ang sinehansabay na kanlungan at kulungan - malinaw ang babala: may hanggahan ang mga waring mapagpalayang pagtatanghal at espasyo kung isasantabi ang mga batayang kondisyong sanhi ng kanilang pag-iral. Mapaniil ang liwanag sa loob dahil gayundin sa labas; ngunit baka sa pagtingala sa liwanag na ito-buhat sa pelikula, mga akdang pampanitikan, at pag-aaral ni Pascualmababaanag ang ilang mapanghawang kasagutan.

GLENN DIAZ is an instructor at the Department of English of Ateneo de Manila University. He has an MA in Creative Writing from the University of the Philippines, Diliman. (gdiaz@ateneo.edu) 